\title{
磷、硼掺杂 $\mathrm{a}-\mathrm{Si}_{1-x} \mathrm{C}_{x}: \mathrm{H}$ 薄膜的 高温 ESR 特性
}

\author{
张仿清 陈光华 程 金 龙 \\ （兰州大学物理系）（兰州大学测试中心）
}

关镜词悬挂键、非晶态半导体、定域化电子、带尾态

\section{一、引吂}

氢化的非晶态硅一碳 $\left(a-\mathrm{Si}_{1-x} \mathrm{C}_{x}: \mathrm{H}\right)$ 膜, 是一种十分重要的非晶态半导体光电材料. 由于 它具有宽的且可变的带隙, 用它作 Pin 型非晶硅太阳电池的窗口材料, 大大地改善了电池 P层的透光性, 使电池的光-电转换效率得到了很大的提高, 因而已受到人们极大的关注. 过去虽 有不少作者研究过有关 $\mathrm{a}^{-} \mathrm{Si}_{1-x} \mathrm{C}_{x}: \mathrm{H}$ 膜的物理特性, 但系统地研究从掺硼到掺磷的样品, 特 别是对用作太阳电池窗口材料的含碳量约为 $20 \%$ 的 $a-S_{i_{1-x}} \mathrm{C}_{x}: H(x=0.2)$ 膜的物理特性却 很少有人报道过. 我们已系统地研究了这种非晶硅碳膜在室温和液复温度下的 ESR 特性 ${ }^{[1-3]}$, 并且首次观察到 $\mathrm{a}-\mathrm{Si}_{\mathrm{t}-x} \mathrm{C}_{x}: \mathrm{H}$ 膜中掺硼的 $\mathrm{ESR}$ 信号.

本文将介绍掺嗍和掺磷的 $a-\mathrm{Si}_{1-x} \mathrm{C}_{x}: \mathrm{H}$ 膜在高温下的 $\mathrm{ESR}$ 特性. ESR 的测量是在高 温热处理的动态过程中进行的, 这样所得的结果更符合于该材料在高温下的实际情况.

\section{二、实 验}

本工作所研究的 $\mathrm{a}-\mathrm{Si}_{\mathrm{t}_{-x}} \mathrm{C}_{x}: \mathrm{H}$ 膜样品, 是用 R.F. 辉光放电法制备的. 通入的气体是 $\mathrm{SiH}_{4}+\mathrm{CH}_{4}$ 的混合气体, 掺杂是分别用 $\mathrm{B}_{2} \mathrm{H}_{3}$ 和 $\mathrm{PH}_{3}$ 气体, 祄底温度约 $250^{\circ} \mathrm{C}$, 系统的预 真空度为 $2.67 \times 10^{-3} \mathrm{~Pa}$ 以下, 气体流星为 $70 \mathrm{sCCM}$, 成膜速度为 $2-3 \AA / \mathrm{s}$, 功率密度为 $\sim 0.4 \mathrm{~W} / \mathrm{cm}^{2}$, 膜厚在 $2 \times 10^{-6} \mathrm{~m}$, 衬底是很薄的 $\mathrm{Al}$ 箔. 将沉积有 $\mathrm{a}-\mathrm{Si}_{1-x} \mathrm{C}_{x}: \mathrm{H}$ 膜的 $\mathrm{Al}$ 箔, 放在 $10-15 \%$ 的稀盐酸中溶去全部的 $\mathrm{Al}$, 经过滤和清洗后低温烘干,即可使用.

掺硼的样品, $\mathrm{B}_{2} \mathrm{H}_{5} /\left[\mathrm{SiH}_{4}+\mathrm{CH}_{4}\right]=10^{-2}$; 掺磷的样品, $\mathrm{PH}_{3} /\left[\mathrm{SiH}_{4}+\mathrm{CH}_{4}\right]=10^{-2}$. 薄 膜的成分是用 XPJ. EPMA (电子探针)和 IR 确定的,碳的含最约为 20 at. \%.

掺杂 $\mathrm{a}-\mathrm{Si}_{1-x} \mathrm{C}_{x}: \mathrm{H}$ 膜的高温 ESR 测量, 是在 ER-200D-SRC 系统的高温谐振腔中完成 的. 这种高温专用谐振腔配有高精度自动加温和测温设备. 在高温测量过程中, 样品一直用 高纯度 $\mathrm{N}_{2}$ 气保护着, 以免样品氧化. 本文所报道的高温 ESR 特性是和通常文献中所报道的 高温 ESR 不同 ${ }^{[4,5]}$, 通常的方法是把样品先经过高温热处理 (退火)后, 再缓慢冷却到实温, 最 后在空温或液氮温度下测量已经过高温热处理后样品的 ESR 特性, 这样获得的实验结果不 能完全反映样品的高温特性, 只能表征该样品的高温退火特性.

本文 1987 年 3 月 14 日收到。

第 15 期

科学通报 1137 


\section{三、结果和讨论}

图 1 和图 2 是我们对未掺杂和掺了嗍磷的 $\mathrm{a}^{-\mathrm{Si}_{1-x}} \mathrm{C}_{x}: \mathrm{H}(x=0.2)$ 膜在高温动态过程中测 量的 ESR 结果.

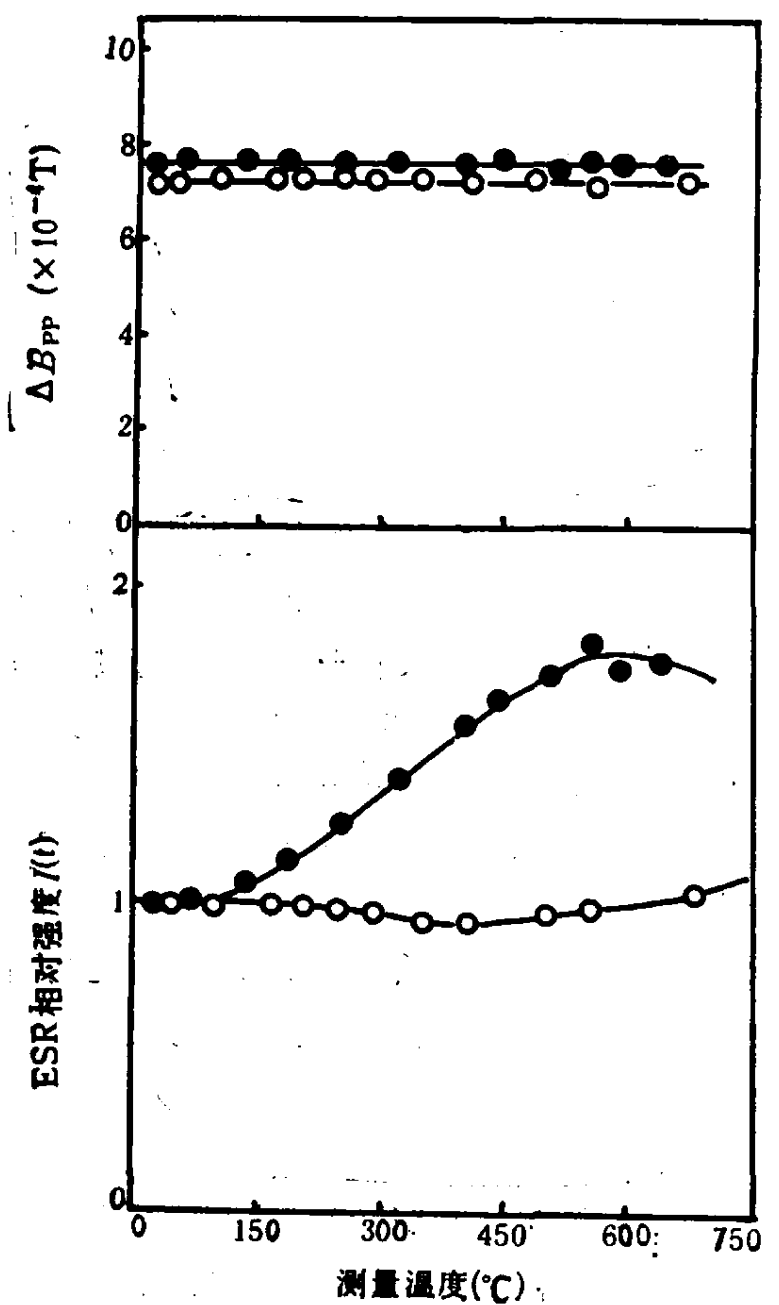

图 1 磷( • 和末掺杂 $(0) a-\mathrm{Si}_{1-\mathrm{x}} \mathrm{C}_{x}: \mathrm{H}(x=0.2)$

膜的 $\Delta B_{p}$ 和 $l(t)$ 跹测量温度的变化曲线

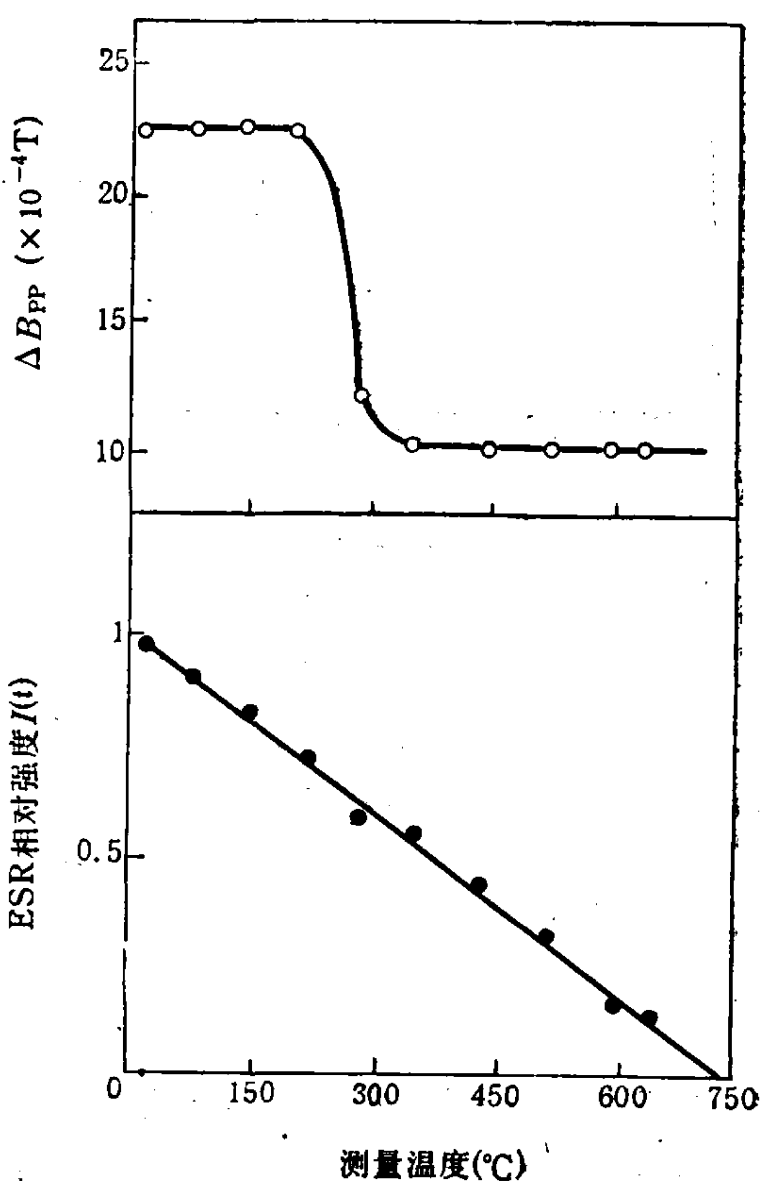

图 2 掺矹的 $\mathrm{a}-\mathrm{Si}_{1-\mathrm{x}} \mathrm{C}_{\mathrm{x}}: \mathrm{H}(x \neq 0.2)$ 膜的 $\Delta B_{n p}$ 和 $I(t)$ 随测量温度 $t$ 的变化曲线

$\Delta B_{p p}$ 是 ESR，吸收峰一峰宽度, $I($ ( ) 为相对于室温下的高温 ESR 吸收强度。对掺硼的 $a-\mathrm{Si}_{1-x} \mathrm{C}_{x}: \mathrm{H}$ 膜来说, 从它的 ESR 波形图(见图 3) 来看, 它主要是一个宽吸收的价带尾态定 域化空穴 ESR 吸收 $\left(g=2.01, \Delta B_{p p}=23 \times 10^{-4} \mathrm{~T}\right)$, 同时还包含了很少量的 $\mathrm{Si}$ 悬挂键 $\left(\mathrm{Si}_{3}^{0}\right)$ 的窄吸收 $\left(g=2.005, \Delta B_{p p}=10 \times 10^{-4} \mathrm{~T}\right)$. 随着温度的升高, 宽带吸收蜂不断地下 降, 在 $300{ }^{\circ} \mathrm{C}$ 以上主要是窄带 $\mathrm{Si}_{3}^{0}$ 的吸收。

下面我们对这种结果作一些试探性的解释.

(1) 对掺砽的 $\mathrm{a}-\mathrm{Si}_{1-x} \mathrm{C}_{x}: \mathrm{H}$ 膜, 随温度的升高,其自旋强度不断地在减小, 在 $300 \mathrm{c}$ 以以下 时 $\Delta B_{p p}$ 为宽带吸收, 在 $300{ }^{\circ} \mathrm{C}$ 以上时 $\Delta B_{p p}$ 转为窄带吸收. 这是由于温度的上升, 使价带 中的电子被激发到价带尾态定域化的空穴能级上，使这种定域化空穴浓度逐渐减少，因而使 $I$ ( $t$ ）也不断下降,这类似于晶态中受主的杂质激发过程. 但超过 $300{ }^{\circ} \mathrm{C}$ 时, 由于此时氢的释放 
使 $\mathrm{Si}$ 的悬搔键 $\left(\mathrm{Si}_{3}^{0}\right)$ 有所增多, 吸收峰就由定域化空穴的宽带吸收转变为 $\mathrm{Si}_{3}^{b}$ 的窄带吸收 (见图 2 和图 3). 又因定域化空穴浓度下降太快， 总的吸收强度 $I(t)$ 还是在减少着.

(2) 对未掺杂的样品,随温度的升高 ESR 信 号的强度变化不大. 在较高温度下因晶格获得部 分能量从亚稳态向稳态过渡，使晶格悬挂键状态 减少，因而 ESR 强度也有所下降。当温度很高 时，一方面发生氢的释放过程使未饱和的 $\mathrm{Si}_{3}^{0}$ 浓 度增多, 另一方面也会出现少量缺陷态 $T_{2}^{+}$和 $T_{2}^{-}-T_{3}^{+}$对 ${ }^{[6]}$,其反应式为

$$
\begin{aligned}
& T_{2}^{0}+T_{3}^{0} \rightarrow T_{2}^{+}+T_{3}^{-}, \\
& T_{2}^{0}+T_{3}^{0} \rightarrow T_{2}^{-}+T_{3}^{+} .
\end{aligned}
$$

根据非晶态半导体化学键原理 ${ }^{[7]}$, (1) 式容易实 现,即产生了 $T_{2}^{+}$中心, 它是对 ESR 有贡献的. (2)式在一般情况下不易实现,但在高温下也可能 成立, 从而产生 $T_{2}^{-}-T_{3}^{+}$缺陷对. 以上所谈的三 种缺陷都是窄带吸收, 且 $g$ 值都接近 $(g \approx 2.005)$, 在高温时都对 ESR 有贡献, 所以此时 $I(t)$ 随温

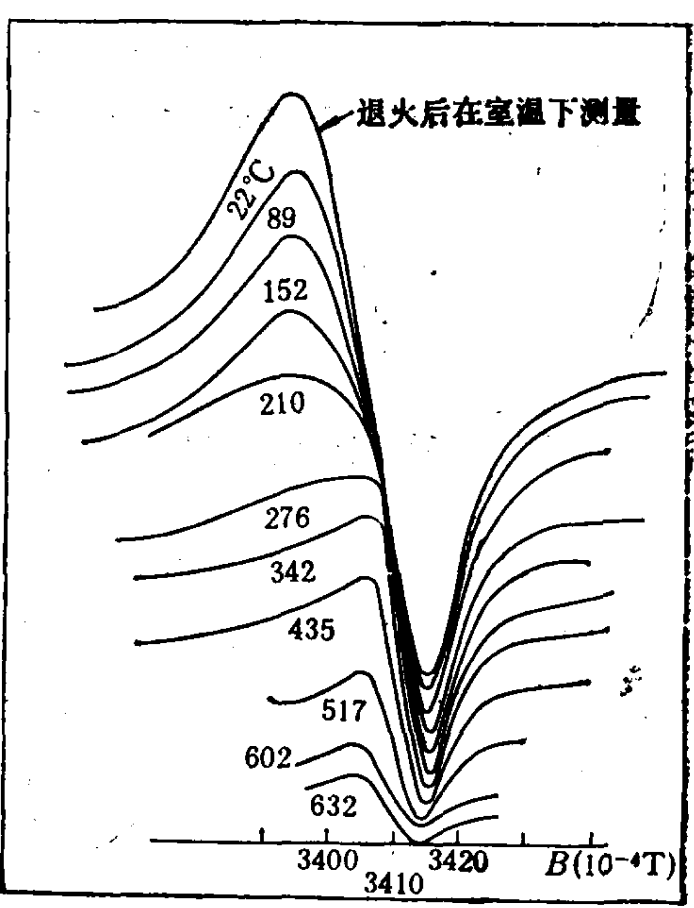

图 3 掺硼的 $2-\mathrm{Si}_{1-x} \mathrm{C}_{x}: \mathrm{H}$ 膜 ESR 波 形随测温度 2 的变化图 度的升高而上升。

（3）对掺磷的样品, 随温度的升高 ESR 强度不断增加. 产生这种 ESR 信号可能有两 种来源。一种是导带尾定域化电子的贡献 ${ }^{[1,2]}$, 它主要是在室温或较低温度下起作用, 在高温下 定域化电子会被激发到导带扩展态去, 反使 ESR 信号减少. 另一种可能是 a-Si:H 中掺 P 后，根据 8-N 法则会产生大量的 $\mathrm{P}_{4}^{+}-\mathrm{Si}_{3}^{-}$缺陷对 ${ }^{(8)}$., 而这种缺陷对本身并没有 ESR 吸收信 兵, 但当温度升高时, 晶格声子的能量会使 $\mathrm{Si}_{3}^{-}$上的一个电子跑到 $\mathrm{P}_{4}^{-}$上去 $\left(E_{F}\right.$ 也会上升 些),结果就会发生

$$
\mathrm{Si}_{3}^{-}-\mathrm{e} \rightarrow \mathrm{Si}_{3}^{0}
$$

反应,而 $\mathrm{Si}_{3}^{0}$ 有窑带的 ESR 吸收峰. 所以随着温度的升高, 虽然导带定域化电子在减少, ESR 强度也在减少, 但是 $\mathrm{P}_{4}^{+}-\mathrm{Si}_{3}^{-}$缺陷对却在迅速地被“解体”, 又使 $\mathrm{Si}_{3}^{0}$ 迅速增多, 因而总的效果 还是 ESR 强度 $I(t)$ 随温度而上升(见图 1). 到 $500^{\circ} \mathrm{C}$ 以后, 因 $\mathrm{P}_{4}^{+}-\mathrm{Si}_{3}^{-}$可能全部解体, ESR 强度不再增加,且因高温引起的自旋弛豫和晶格弛豫而使 ESR 吸收强度 $I(t)$ 有适当减少。

\section{考文献}

[1] Chen, G. H., Morimoto, A., Kumeda, M. and Shimizu, T., Phys. Stat. Sol, (a) 89 (1985), k181.

[ 2 ] Chen Guanghua, Zhang Fangqing, Xu Xixiang, Morimoto, A., Kumeda, M. and Shimizu, T., Chinese Physics, 6 (1986)，918. 陈光华、张仿清、徐希翔、森本章治、久米田秢、清水立生，物理学报，35(1986),517.

[3] 陈光华、张仿清、徐希翔、清水立生,半导体学报, 7(1986), 539 .

[4] Hasegawa, S. and Yazaki, S., Thin Solid Films; 55(1978), 15.

[5] Kumeda, M., Jinno, Y., Watanabe, 1. and Shimizu, T., Solid Statc Commun., 23(1977), 833.

[6] Wis, Y. and Stesmans, A., Phys. Rev., B-33 (1986), 5046.

[7] 清水论生,固体物理(日),15(1980), 408 .

[8] Robertson, J., Phys. Rev., B-31(1985), 3817; J. Non-iryst. Solids., 77 \& 78 (1985), 37. 